procedural sICH was significantly lower in DO group compared to PO group ( $0 \%$ vs. $6 \%, \mathrm{p}<0.05)$; vessel recanalization (TICI $\geq 2$ b) was not significantly different between DO and PO. However, procedure time was significantly shorter in the DO group (45 min vs. $52 \mathrm{~min}, \mathrm{p}=0.05)$ and the mean number of passes were lower in the DO group (1.5 vs. 1.8, $\mathrm{p}=0.01)$. Mortality rate and good functional outcome was not significantly different between both groups. On multivariate analysis, DO group had a significantly shorter length of stay by about two days, compared to PO, when controlling for confounders (coef: -2 days, 95\%CI: -4 to 0 days, $p=0.05$ ). Subjects with PO were over five times more likely to have a good TICI score compared to DO group (OR: 5.69, 95\% CI: $1.22-26.5, \mathrm{p}<0.05)$. There was no significant difference in peri-procedural and post-procedural complications, a good functional outcome at three months, and mortality between both groups.

Conclusion Mechanical thrombectomy procedure for distal circulation strokes is as effective and safe as a proximal group. Though distal vessels supply smaller brain area, however when symptoms are pronounced the benefit of the procedure outweighs the risks.

Disclosures A. Sweid: None. J. Head: None. S. Tjoumakaris: None. V. Xu: None. K. Shivashankar: None. T. Alexander: None. M. Gooch: None. N. Herial: None. R. Rosenwasser: None. P. Jabbour: None.

\section{E-119 FEASIBILITY FOR EXTRACRANIAL CAROTID ARTERY STENTING IN ACUTE ISCHEMIC STROKE PATIENTS AFTER IV-TPA TREATMENT}

J Seo*, E Kim. Neurology, Inje University colleage of Medicine, Busan, Korea, republic of

10.1136/neurintsurg-2019-SNIS.194

Purpose The clinical benefit of endovascular thrombectomy (EVT) after intravenous recombinant tissue plasminogen activator (IV rt-PA) has been demonstrated in several studies. Near $10 \%$ of acute ischemic stroke (AIS) patients are accompanied with carotid artery atherosclerotic occlusion. The MR Clean study in the Netherlands reported that acute carotid artery stenting (CAS) was performed in 30 patients in the intervention group (12.9\%). However, there is no evidence of the benefit for emergent CAS in AIS patients, and there are no clear guidelines for specific medications as an adjunctive therapy within 24 hours of IV rt-PA.

Methods We analyzed single-center registry data retrospectively for 2006 to 2018. Emergent CAS during EVT was performed in select patients according to the operator's decision. Most patients for emergent CAS was not used to antithrombotic medications routinely within 24 hours of IV rt-PA. Only some complicated cases were used intra-arterial or oral antithrombotics within 24 hours of IV rt-PA. Recanalization results were assessed by TICI grade immediately after the procedure. The clinical outcome was evaluated using the 3 months modified Rankin Scale.

Results Among 177 consecutive patients with AIS treated with rt-PA and EVT from 2006 to 2018, twenty-three(12.9\%) patients underwent CAS as an EVT. Eighteen patients $(78.3 \%)$ were accompanied with tandem occlusion. Median age was 68. Median NIHSS was 13. During the emergent carotid artery stenting, pre-stent balloon angioplasty was performed in 20 patients. Carotid Wallstent (Boston Scientific, Natick, USA) was used in all patients who treated with emergency CAS. Successful installation of CAS was achieved in 22 patients (95.6\%). One case of CAS was failed. Two patients were installed multiple carotid stent for carotid dissection. And embolic protection device was used in 13 patients.However, four cases of CAS experienced thromboembolic complications including in-stent thrombosis and distal migration of emboli. In those cases, IA glycoprotein IIb/IIIa infused as a rescue method, and early antiplatelet within 24 hours of rt-PA was administered in one patient.Final successful recanalization (mTICI 2b-3) was achieved in 16 patients (69.5\%). Eleven patients $(47.8 \%)$ had a good functional outcome (modified mRS 0-2). Intracranial hemorrhage was occurred in 4 cases. The mortality case was two. The accompanying tandem occlusion was $61 \%$.

Conclusions In this analysis, successful rate of emergency CAS and intracranial EVT after IV rt-PA was high as $95.6 \%$ and $69.5 \%$. The good functional outcomes (mRS 0-2) were $46.7 \%$ in patients treated with emergency CAS and the overall clinical outcome would be acceptable.

Disclosures J. Seo: None. E. Kim: None.

\section{E-120 VECTA 071 AND 074 LARGE BORE ASPIRATION CATHETER: INITIAL MULTI-CENTER EXPERIENCE}

${ }^{1} \mathrm{~S}$ Satti*, ${ }^{1} \mathrm{~T}$ Sivapatham, ${ }^{2} \mathrm{E}$ Almallouhi, ${ }^{1} \mathrm{~T}$ Eden, ${ }^{2} \mathrm{~A}$ Spiotta. ${ }^{1}$ Neurointerventional Surgery, Christiana Care Health System, Newark, DE; ${ }^{2}$ Neurosurgery and Endovascular Neurosurgery, Medical University of South Carolina, Charleston, SC

\subsection{6/neurintsurg-2019-SNIS.195}

Background and objective New large bore aspiration catheters are being used as first line therapy for mechanical thrombectomy in patients with emergent large vessel occlusions. We present the largest combined experience with the new Stryker Neurovascular Vecta $071 / 074$ aspiration catheters. Vecta 074 represents the largest available aspiration catheter in the U.S. We sought to assess the safety, efficacy, direct aspiration first pass success, need for rescue with stent retriever, and clinical improvement.

Methods Multi-institution retrospective review of all Vecta aspiration catheter cases performed at 2 comprehensive stroke centers between January 1, 2019 and March 25, 2019 yielded 26 patients. Key metrics were recorded including pre-procedure ASPECTS score, presenting NIHSS score, last seen normal to groin access, groin access to first device deployment, time to recanalization, revascularization TICI grading, thrombectomy technique (successful direct aspiration, need for rescue stent retriever and number of passes), and outcomes (NIHSS at discharge, rate of symptomatic intracranial hemorrhage sICH, and death prior to discharge).

Results Eighteen 071 and six 074 Vecta aspiration catheters were used (figures.1/2). The average pre-procedure ASPECTS, NIHSS, and time from last seen normal were 8, 17.7, and 5.3 hours, respectively. Time from vascular access to first device delivery was 25.9 minutes. Direct aspiration was attempted as first line technique in all cases and was successful in $72 \%$ of cases. $28 \%$ of cases required use of a stent retriever to deliver the aspiration catheter (grappling hook technique-figure.3), or for rescue thrombectomy (direct aspiration unsuccessful or to target residual small, distal emboli). TICI 3 revascularization was achieved in $46 \%$, TICI $2 \mathrm{C}$ in $23 \%$, TICI $2 \mathrm{~B}$ in $26 \%$, and $5 \%$ with unsuccessful TICI $2 \mathrm{~A}$ revascularization. The average NIHSS on discharge was 6.1 with two patients expiring prior 


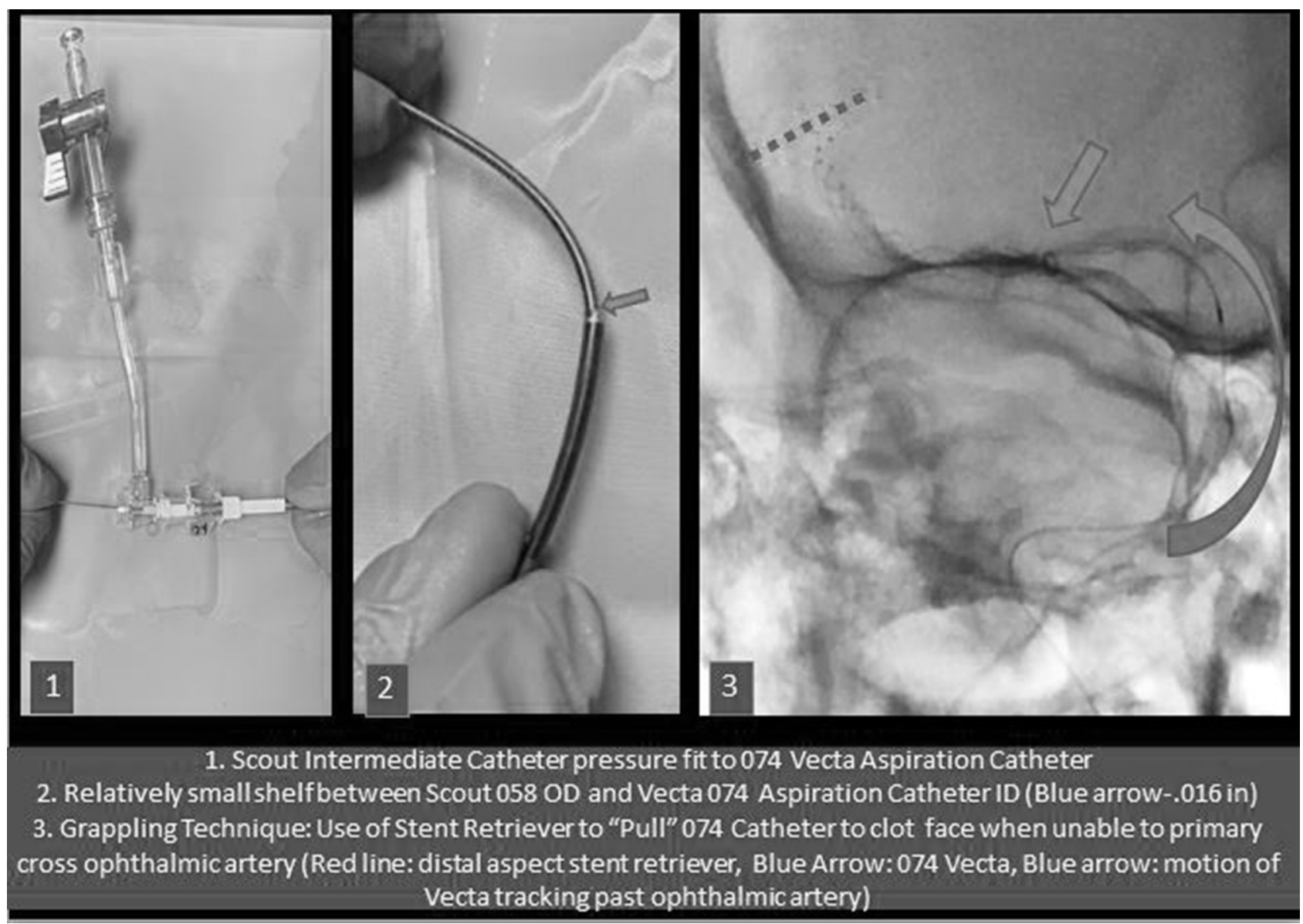

\section{Abstract E-120 Figure 1}

to discharge (withdrawal of care in two patients with basilar artery occlusion). Complications included one intracranial/extradural non-flow limiting dissection and one sICH. Limitations included: Small sample size, retrospective review, variation in data collection due to differences in institutional records and short time to follow up.

Conclusion Initial experience with the new Stryker Neurovascular Vecta 071/074 aspiration catheters suggests that high TICI 2C/3 reperfusion rates can safely be obtained using direct aspiration in a majority of cases. Stent retrievers can be reserved for cases where grappling hook technique is necessary to deliver the aspiration catheter to the clot or for rescue thrombectomy.

Disclosures S. Satti: 2; C; Stryker Neurovascular, Medtronic Neurovascular, Penumbra Neurovascular, Cerenovus Neurovascular, Terumo. T. Sivapatham: 2; C; Penumbra Neurovascular. E. Almallouhi: None. T. Eden: None. A. Spiotta: 2; C; Minnetronix Neurovascular, Cerenovus Neurovascular, Penumbura Neurovascular.

\section{E-121 SINGLE CENTER EXPERIENCE WITH A NOVEL FDA- APPROVED PERCUTANEOUS FEMORAL ARTERY CLOSURE DEVICE (CELT ACD ${ }^{\circledR}$ ) IN NEUROVASCULAR PROCEDURES}

W Htun, N Patel, R Rabinovich, S Newman, A Wakhloo*. Neurointerventional Radiology, Beth Israel Lahey Health, TUFTS Univ Med School, Burlington, MA

10.1136/neurintsurg-2019-SNIS. 196
Purpose Celt $\mathrm{ACD}^{\circledR}$ (Vasorum Ltd. Dublin, Ireland) is a unique percutaneously applied vascular closure device that achieves hemostasis through the delivery of a stainless steel (316 LVM) plug which is anchored on both sides of the arterial wall. The extendable wings of the implant provide an immediate vascular closure. We studied the safety and efficacy profile of the Celt in our neurodiagnostic and interventional cases.

Patients and method After obtaining a local IRB approval, Celt was used in all consecutive neurodiagnostic and interventional cases from 2018 to 2019. Patients presented with or without a systemic thrombolytic agent or a dual antiplatelet therapy. A 5, 6 and $7 \mathrm{~F}$ Celt system was available for closure. The $7 \mathrm{~F}$ device can be used for a femoral sheath size up to 9 F. Recorded data were analyzed for an immediate failure to close the access site, early or delayed migration of the implant, pain or delayed neuropathy, infection, early or delayed hematomas and other unforeseeable complications at the puncture site.

Results Celt was applied in 271 neuro-endovascular procedures in a total of 204 patients (M: F ratio 1.4: 1; age range 24 - 93 years). Majority of subjects (72\%) presented either on systemic thrombolytic agents (tPA or Heparin) or were placed on a dual antiplatelet agent prior to the procedure. The closure device was used for a right femoral artery access in $76.4 \%$, left femoral artery in $19.9 \%$, right femoral vein in $1.48 \%$ and left femoral vein in $2.2 \%$ of the cases. The most common implant used was a $5 \mathrm{~F}$ system $(55.7 \%)$, followed by a $6 \mathrm{~F}(26.19 \%)$ and a $7 \mathrm{~F}(18.08 \%)$ system. Three (1.4\%) major complications and 18 (8.8)\% minor 